\title{
An alternative method for evaluating the coherence length of a laser diode
}

Po-Jen Hsieh, Zhi-Cheng Jian, Der-Chin Su

Po-Jen Hsieh, Zhi-Cheng Jian, Der-Chin Su, "An alternative method for evaluating the coherence length of a laser diode," Proc. SPIE 9664, Ninth International Topical Meeting on Education and Training in Optics and Photonics, 96642N (24 October 2005); doi: 10.1117/12.2207729

SPIE Event: Ninth International Topical Meeting on Education and Training in Optics and Photonics, 2005, Marseille, France 


\title{
Ref ETOP066
}

\section{An alternative method for evaluating the coherence length of a laser diode}

\author{
Po-Jen Hsieh, Zhi-Cheng Jian and Der-Chin Su
}

Department of Photonics \& Institute of Electro-Optical Engineering, National Chiao-Tung University, 1001 Ta Hsueh Road, Hsin-Chu 30050, Taiwan, R. O. C.

\begin{abstract}
Let a light beam coming from a diode pass through a polarizer and an electro-optical modulator driven a saw tooth voltage signal, then a slight frequency difference between its two linearly orthogonal polarized components occurs. The light beam enters a modified Michelson interferometer, and the interference signal is sent to an oscilloscope. Because the interference signal is a periodic function of time, it is easier than the conventional one to evaluate the contrast. When the contrast is zero, the associated optical path difference is its coherence length.
\end{abstract}

\section{Keywords}

coherence length, Michelson interferometer, Jones vectors, Optical path difference.

\section{Summary}

The schematic diagram of this method is shown in Fig. 1. The light beam coming from a laser diode LD passing through a polarizer $P$ and an electro-optic modulator $E O$ incidents on a beam splitter BS. The transmitted light enters a modified Michelson interferometer ${ }^{[1]}$ and is divided into two parts by a polarization beam splitter PBS: the reflected s-polarization light and the transmitted $\mathrm{p}$-polarization light. The former is normally reflected by a mirror $\mathrm{M}_{1}$, and then is reflected by the PBS and the BS again. And it passes through an analyzer AN and is detected by a photo detector PD. It acts the reference light in this interferometer. The latter is normally reflected by another mirror $M_{2}$ and returns along its original path. After being reflected by the $B S$, it passes through the AN and also enters the PD. It acts as the test light in the modified Michelson interferometer. If the amplitudes of the reference light and the test light are $E_{r}$ and $E_{t}, P D$ measures the interference intensity of $E_{r}$ and $E_{t}$, that is, $l=\left|E_{r}+E_{t}\right|^{2}$.

For convenience, the $+z$ axis is chosen along the propagation direction, the $x$-axis is along the horizontal direction and only the light of the wavelength $\lambda_{i}$ is considered. Let the transmission axis of the $P$ be at $45^{\circ}$ with respect o the x-axis, then the Jone vector ${ }^{[2]}$ of the light incidenting on the BS can be written as

$E=\frac{1}{\sqrt{2}}\left(\begin{array}{l}1 \\ 1\end{array}\right)$.

If the fast axis of the EO is along the x-axis, and an external saw tooth voltage signal with frequency $f$ and amplitude $V$ is applied to the $E O$, then the retardation produced by the EO can be expressed as ${ }^{[3,4]}$

$$
=2 \pi \frac{V}{V_{i}} f t, \quad \Gamma
$$

where $V_{i}$ is the associated half-wave voltage of the EO at $\lambda_{i}$. And if the transmission axis of the $\mathrm{AN}$ is $45^{\circ}$ with respect to the $\mathrm{x}$-axis, then we have

$E_{r}=\frac{1}{2 \sqrt{2}}\left(\begin{array}{l}1 \\ 1\end{array}\right) e^{-i \frac{\Gamma}{2}}$,

and 
$E_{t}=\frac{1}{2 \sqrt{2}}\left(\begin{array}{l}1 \\ 1\end{array}\right) e^{i\left(\frac{\Gamma}{2}+\phi_{i}\right)}$,

where $\phi_{i}$ is the phase difference being corresponding to the optical path difference between the test light and the reference light, i.e.,

$$
\phi_{i}=4 \pi \frac{\left(l_{2}-l_{1}\right)}{\lambda_{i}} .
$$

Therefore, the interference intensity is given by

$$
I_{i}=\left|E_{r}+E_{t}\right|^{2}=\frac{1}{2}\left[1+\cos \left(\Gamma+\phi_{i}\right)\right]=\frac{1}{2}\left[1+\cos \left(2 \pi \frac{V}{V_{i}} f t+\phi_{i}\right)\right] .
$$

Because the light coming from a laser diode contains many continuous spectrums, the interference intensity measured by the PD can be expressed as

$I \cong \sum_{i=1}^{n} I_{i}=\frac{1}{2} \sum_{i=1}^{n} a_{i}\left[1+\cos \left(2 \pi \frac{V}{V_{i}} f t+\phi_{i}\right)\right]$,

where $n$ is an integer, and $a_{i}$ is the bias intensity of wavelength $\lambda_{i}$. From Eq. (7), it is obvious that we can obtain the summation signal of many cosine signals on the oscilloscope OSC. Then, moving $\mathrm{M}_{2}$ in steps until the contrast of the signal on the OSC is nearly equivalent to zero. At the time, the optical path difference between the test light and the reference light is the coherence length.

In order to show the validity of this method, a laser diode (HHL6720G) manufactured by Japan Hitachi Ltd. is tested. It operated at $25{ }^{\circ} \mathrm{C}$ with electric current $35 \mathrm{~mA}$ and its central wavelength is $670.57 \mathrm{~nm}$. An EO modulator (4002) fabricated by New Focus, with half-wave voltage $220 \mathrm{~V}$ at $670.57 \mathrm{~nm}$, is used in this test. A saw tooth signal, with frequency $2 \mathrm{kHz}$ and amplitude

$220 \mathrm{~V}$, is applied to the EO modulator. Fig. 2 are the interference signal on the OSC as the optical path difference is about (a) zeromm, (b) $27 \mathrm{~mm}$, (c) $42 \mathrm{~mm}$, respectively. According Fig. 2, we obtain its coherence length is about $42 \mathrm{~mm}$. In addition, we also measure its coherence length with the conventional Michelson interferometer. We obtain the same result.

The half-voltage of the EO is varied as the wavelength is varied ${ }^{[5]}$. In our test, the tested LD has narrow spectral bandwidth, the condition $V \cong V_{i}$ exists in Eq. (7). So our measured result has the same result as the conventional method. If a light source with wide spectral bandwidth or several separate spectral lines ${ }^{[6]}$, is tested, the half-voltage is different for different wavelength. Consequently, the measured result with this method will be smaller than that of the conventional method. We also measure two He-Ne lasers (GLG 5369 and GLG 5730) manufactured by Japan NEC Ltd. The half-voltage $146.4 \mathrm{~V}$ at $632.8 \mathrm{~nm}$ is applied to the EO. Their measured results are

$256 \mathrm{~mm}$ and $378 \mathrm{~mm}$ (with this method), and $305 \mathrm{~mm}$ and $450 \mathrm{~mm}$ (with the conventional method), respectively. Therefore this method is suitable only for the light source with narrow spectral bandwidth. In addition, either mirror does not move as the contrast of the interference signal is monitored in the process of this method, so this method has some merits such as high stable signal, easy operation, and high accuracy.

\section{Acknowledgement}

This study was supported in part by the National Science Council, Taiwan, under contract NSC 93-2215-E-009-021. 


\section{References}

[1] P. Hariharan, "Optical interferometery", Academic Press, $2^{\text {nd }}$ Edition., 2003.

[2] A. Yariv and P. Yeh, "Optical waves in crystals", John Wiley and Sons, Inc., N. Y., Chap. $5,1984$.

[3] D. C. Su, M. H. Chiu, and C. D. Chen, "Simple two-frequency laser", Precision Engineering, 18, pp. 161-163, May 1996

[4] D. C. Su, M. H. Chiu, and C. D. Chen, "A heterodyne interferometer using an electro-optic modulator for measuring small displacements", J. Optics, Vol. 27, pp. 19-23, 1996.

[5] A. Yariv and P. Yeh, "Optical waves in crystals", John Wiley and Sons, Inc., N. Y., Chap. 7, 1984.

[6] O. Svelto and D. C. Hanna, "Principles of Lasers", Plenum Press, N. Y., $3^{\text {rd }}$ Edition, 1989.

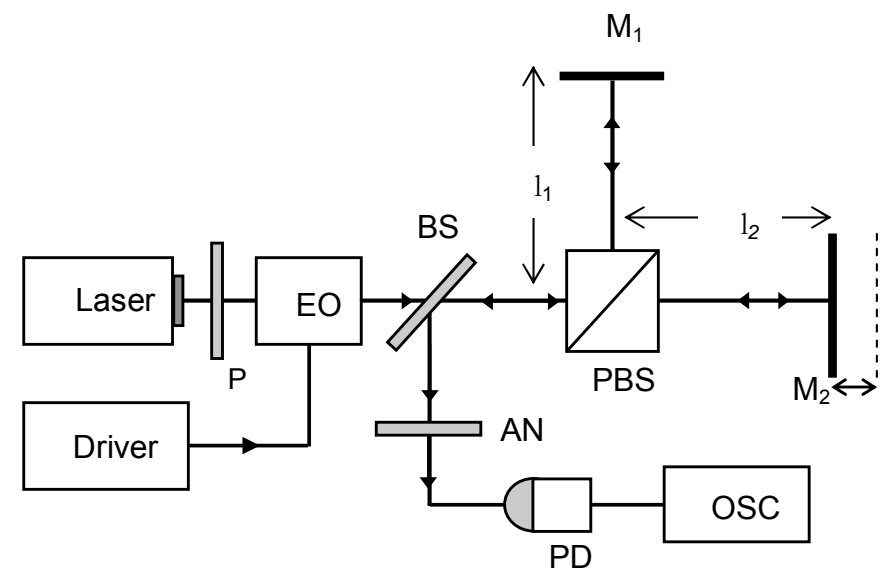

Fig. 1 The schematic diagram of this method for measuring the coherence length of a laser diode. P: polarizer; EO: electro-optic modulator; BS: beam splitter; PBS: polarization beam splitter; M: mirror; AN: analyzer; PD: photo detector; OSC: oscilloscope.

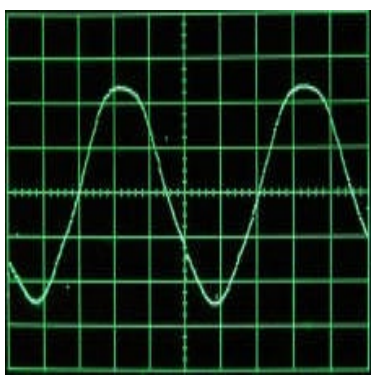

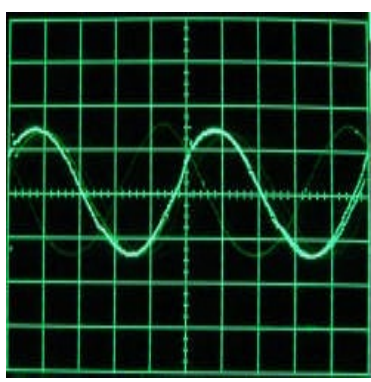

(a)

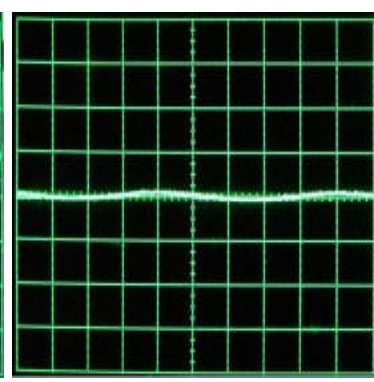

(b)

Fig. 2 The interference signals on the OSC as the optical path difference is about (a) $0 \mathrm{~mm}$, (b) $27 \mathrm{~mm}$, and (c) $42 \mathrm{~mm}$, respectively. 